\title{
The effects of PPARy agonist rosiglitazone on neointimal hyperplasia in rabbit carotid anastomosis model
}

\author{
Mehmet Guzeloglu*, Buket Reel², Soner Atmaca ${ }^{3}$, Alper Bagrıyanık ${ }^{3}$ and Eyup Hazan ${ }^{1}$
}

\begin{abstract}
Background: Neointimal hyperplasia involving smooth muscle cell (SMC) proliferation, migration and extracellular matrix (ECM) degradation is an important component of atherosclerosis. It develops as a response to vascular injury after balloon angioplasty and vascular graft placement. Matrix metalloproteinases (MMPs) induce SMC proliferation, migration and contribute to intimal hyperplasia by degrading ECM. PPARY agonists inhibit SMC proliferation, migration and lesion formation. In this study, we aimed to investigate the effects of PPARY agonist rosiglitazone on neointimal hyperplasia and gelatinase (MMP-2 and MMP-9) expressions in rabbit carotid anastomosis model.
\end{abstract}

Methods: New Zealand white rabbits $(n=13,2.7-3.2 \mathrm{~kg})$ were divided into placebo and treatment groups. Right carotid artery (CA) was transected and both ends were anastomosed. Treatment group $(n=6)$ received rosiglitazone (3 mg/kg/day/p.o.) and placebo group $(n=7)$ received PBS (phosphate buffered saline, $2.5 \mathrm{ml} / \mathrm{kg} /$ day $/$ p.o.) for 4 weeks postoperatively. After the sacrification, right and left CAs were isolated. Morphometric analyses and immunohistochemical examinations for gelatinases were performed.

Results: Intimal area $\left(0.055 \pm 0.005\right.$ control vs $0.291 \pm 0.020 \mu \mathrm{m}^{2}$ anastomosed, $\left.\mathrm{p}<0,05\right)$ and index $(0.117 \pm 0.002$ control vs $0.574 \pm 0.013$ anastomosed, $p<0,01)$ significantly increased in anastomosed arteries compared to control arteries from placebo group. However, in rosiglitazone-treated group, intimal area $(0.291 \pm 0.020$ PBS vs $0.143 \pm 0.027$ rosiglitazone, $p<0,05)$ and index $(0.574 \pm 0.013$ PBS vs $0.263 \pm 0.0078$ rosiglitazone, $p<0,01)$ significantly decreased. Furthermore, gelatinase immunopositivity was found to have significantly increased in anastomosed arteries from placebo group and decreased with rosiglitazone treatment.

Conclusions: These results suggest that rosiglitazone may prevent neointimal hyperplasia, which is the most important factor involved in late graft failure, by inhibiting gelatinase enzyme expression.

Keywords: Neointima, Rosiglitazone, Matrix metalloproteinases (MMPs), Rabbit

\section{Background}

Neointimal hyperplasia has a major role in early restenosis after surgical interventions such as surgical revascularisation, percutan transluminal angioplasty (PTA) and stenting [1,2]. It is an early and essential step in the pathogenesis of atherosclerosis and restenosisis. This step is characterised by extracellular matrix (ECM) degradation, and medial vascular smooth muscle cell migration to intima and their proliferation.

\footnotetext{
* Correspondence: mehmetguzeloglu@hotmail.com

${ }^{1}$ Department of Cardiovascular Surgery, Faculty of Medicine, İzmir University, Izmir, Turkey

Full list of author information is available at the end of the article
}

Matrix metalloproteases (MMPs) are a family of zincdependent enzymes which induce smooth muscle proliferation and migration by degrading ECM and contribute to intimal hyperplasia, inflamation and plaque rupture [3]. Therefore, inhibition of MMPs may be a crucial strategy to reduce the development of intimal hyperplasia.

Peroxisome proliferator-activated receptors (PPARs) are ligand-activated nuclear receptor family. The three PPAR isotypes, PPAR- $\alpha$, PPAR- $\delta$ and PPAR- $\gamma$ modulate the function of many target genes and participate in the regulation of vital processes such as inflammation, cell growth, proliferation, migration and differentiation [4-6].

PPAR $\gamma$ is expressed predominantly in adipose tissue and it is also present in endothelial cells, smooth muscle

\section{Biomed Central}


cells and monocytes/macrophages [7]. Recent studies demonstrated that the activation of PPAR $\gamma$ inhibited MMP expression in cultured macrophages and hypercholesterolemic mice $[8,9]$. This effect contribute to their antiproliferative effect on SMCs. Indeed, PPAR $\gamma$ agonists are shown to decrease migration and proliferation of human and rat vascular SMCs [5]. Similarly, it was reported that dominant-negative loss of PPAR $\gamma$ function enhances SMC proliferation, migration, and vascular remodeling in isolated transgenic mice SMCs [10].

Thiazolidinediones (TZDs), which are widely used in the treatment of type II diabetics as insulin sensitizers, are selective activators of PPAR $\gamma$ [11]. Rosiglitazone, a synthetic PPARY agonist, was reported to inhibit neointimal hyperplasia in rats after balloon injury, and to reduce SMC proliferation in rat SMC culture [5,12,13]. Moreover, clinical studies revealed that PPAR $\gamma$ agonists, rosiglitazone and pioglitazone inhibit development of neointimal hyperplasia and restenosis after percutaneus coronary intervention in diabetic coronary artery patients $[14,15]$.

Although vascular protective effects of rosiglitazone in some atherosclerosis models and cell culture are known, its effects on proinflammatory gelatinase $\mathrm{A}$ and $\mathrm{B}$ enzymes (MMP-2 and MMP-9) related to atherosclerotic process were not thoroughly understood.

In the light of the collected data, the purpose of the present study was to investigate the effects of PPAR $\gamma$ agonist rosiglitazone on neointimal hyperplasia process and gelatinase expressions in rabbit carotid anastomosis model.

\section{Methods}

\section{Animals}

This study was approved by the Local Ethics Committee of Dokuz Eylul University, School of Medicine. All animals received care in compliance with the principles of laboratory animal care formulated by the National Society for Medical Research and the Guide for the Care and Use of Laboratory Animals. In this study, New Zealand white rabbits of either sex $(n=13 ; 2,7-3,2 \mathrm{~kg})$ were used. Rabbits were randomly divided into two groups as placebo and treatment groups. Throughout the 4-week treatment period, rabbits from treatment group $(n=6)$ received rosiglitazone ( $3 \mathrm{mg} / \mathrm{kg} /$ day, p.o.) postoperatively [16]. Rabbits from placebo group $(n=7)$ received only the vehicle (PBS; phosphate buffered saline) $(2.5 \mathrm{ml} / \mathrm{kg} /$ day, p.o.) for the same period. Throughout the 4-week treatment period each rabbit was kept in a separate cage and allowed to access to regular diet (standard rabbit chow and tap water ad libitum). All animals tolerated drug treatment well. The treatment protocol did not affect survival rate and body weight of animals from both groups (data not shown).

\section{Surgical procedures}

Rabbits were anesthetized with intramuscular xylazine (3 mg/kg) and ketamine (50 mg/kg). All procedures were performed by the same surgeon using a 3.5X surgical telescope (US PAT NO: 3273456, Designs for Vision, Ronkonkoma, NY, USA). An oblique cervical incision was made and the right carotid artery was surgically accessed. Rabbits were heparinised with $100 \mathrm{IU} / \mathrm{kg}$ heparin. Three minutes after heparisination, distal and proximal parts were clamped and the right carotid artery was transected. Subsequently, both ends of right carotid artery were anastomosed in an end-to-end form using an $8 / 0$ polypropylene suture [17]. Then, a similar incision was made on the left contralateral carotid artery and a segment from the left carotid artery which constituted control groups (Rosiglitazone control and PBS control) was resected. Afterwards, the carotid arteries were returned to their original positions and the anatomic layers were closed properly. Following the operation xylazine was given intramusculary to the rabbits for analgesia.

On the $28^{\text {th }}$ postoperative day, the rabbits were sacrificed using an over dose of sodium pentobarbital (150 mg/kg). Subsequently, the anastomosed right carotid artery segment and then a segment from the contralateral intact carotid artery as control artery were isolated and removed. Each segment was put into a tube containing $10 \%$ neutral buffered formalin immediately for histological examination.

\section{Histological evaluations \\ Morphometry}

After sacrification of the rabbits, tissue samples were prepared for analysis by light microscopy and immunohistochemistry. Tissues were fixed in $10 \%$ neutral formalin solution for 24-48 hrs. Then the samples were embedded in paraffin and serial sections were taken onto glass slides as in $5 \mu \mathrm{m}$ thickness. After staining by Orcein Light Green, they were mounted by using Entellan mounting medium. Samples were examined under a light microscope (Olympus BH2, Tokyo, Japan) and images were transferred to the computer using a digital video camera (Olympus DP71, Tokyo, Japan). The light microscope was connected to an image capture system in all groups and measurements. Multiple fields (up to 5) were selected from each section of each sample in a systematic random pattern and images were taken and analyzed by using software (Image Tool, Uthscsa-version 3.00 for Windows, analysis system). The images were processed and measurements were taken in crosssections. Briefly, intima and media areas $\left(\mu \mathrm{m}^{2}\right)$ were meausured using computer-assisted image analyzer system. Additionally, ratios of intima/media (index values) were calculated. 


\section{Immunohistochemistry}

Five micrometer sample sections were incubated at $60^{\circ} \mathrm{C}$ overnight then deparaffinised in xylene for $30 \mathrm{~min}$. After rehydrating by using a decreasing series of ethanols, sections were washed in distilled water for $10 \mathrm{~min}$. Then they were heated with $10 \mathrm{mM}$ citrate buffer (Cat \# AP9003-125 Labvision) for five minutes, to unmask antigens. After washing in deionized water for three times with two minutes period, sections were delineated using a Dako pen (Dako, Glostrup, Denmark), In order to inhibit endogenous peroxidase activity samples were incubated in 3\% $\mathrm{H} 2 \mathrm{O} 2$ for $5 \mathrm{~min}$. After blocking samples with serum solution for 30 minutes, sections were incubated with primary antibody in a humid chamber for $18 \mathrm{~h}$ at $+4^{\circ} \mathrm{C}$. Anti-MMP-2 (cat. \# MAB 3308, Milipore, MA, ABD), anti-MMP-9 (Cat. \# MAB 3309, Milipore, MA, ABD) primary antibodies were used. Samples were then incubated with anti-mouse secondary antibodies conjugated with biotin and with streptavidin conjugated to horseradish peroxidase for $30 \mathrm{~min}$ each prepared according to kit instructions (Cat \# 85-9043, HistostainPlus Bulk Kit Broad Spectrum, Invitrogen, Camarillo, California, USA). They were finally incubated with 3,30 diaminobenzidine hydrochloride (DAB) (Cat \# 1718096, Roche, Oenzberg, Germany), and nuclei were counterstained with Mayer's hematoxylin. All dilutions and washing steps were performed by using phosphate buffered saline (PBS), pH 7.4. Sections were dehydrated through a graded ethanol series, cleared in xylene, mounted in Entellan (Cat \# 107961, Merck, Darmstadt, Germany) and analyzed using a light microscope. Appropriate positive controls were also stained.

\section{Statistical analysis}

Statistical analysis of morphometric data were performed for drug treatments (two levels, rosiglitazone or placebo) as between rabbit factor; and anastomosis (two levels, present or not) as within rabbit factor with factorial analysis of variance (ANOVA). If there were interactions between the factors in ANOVA, Wilcoxon signed ranks test and Mann Whitney $U$ test were used for paired and unpaired comparisons respectively.

Chi-square test was used to evaluate the statistical difference of immunohistochemical data between rosiglitazone and placebo group. Statistical analyses of paired data (anastomosed vs control) from immunoscoring were performed with Wilcoxon signed ranks test. Shown are means \pm SEM. $\mathrm{n}$ indicates the number of animals. $\mathrm{p}<0.05$ was considered statistically significant.

\section{Results}

Morphometry

Intense intimal hyperplasia was observed in anastomosed right arteries as compared to those in control left arteries in placebo group as a result of morphometric examinations.

The intimal cross-sectional area $\left(0.055 \pm 0.005 \mu \mathrm{m}^{2}\right.$ control left vs $0.291 \pm 0.020 \mu \mathrm{m}^{2}$ anastomosed right arteries) $(\mathrm{p}<0.05)$ and the ratio of intimal area to medial area (index) $(0.117 \pm 0.002$ control vs $0.574 \pm 0.013$ anastomosed right arteries) $(\mathrm{p}<0.05)$ significantly increased in anastomosed right arteries as compared to those in control left arteries in placebo group (Figure 1; Figure 2; Figure 3).

However, rosiglitazone treatment significantly decreased the neointimal hyperplasia $\left(0.291 \pm 0.020 \mu \mathrm{m}^{2}\right.$ PBS group vs $0.143 \pm 0.027 \mu^{2}$ rosiglitazone group) $(\mathrm{p}<0.01)$ and carotid artery intima/media index $(0.574 \pm 0.013$ PBS group vs $0.263 \pm 0.0078$ rosiglitazone group) $(\mathrm{p}<0.01)$ in anastomosed right arteries (Figure 2, Figure 4, Figure 3 ).

Medial area $\left(0.508 \pm 0.032 \mu \mathrm{m}^{2}\right.$ control left vs $0.468 \pm$ $0.043 \mu \mathrm{m}^{2}$ anastomosed right arteries) $(\mathrm{p}=0.075)$ and luminal area $\left(0.444 \pm 0.042 \mu \mathrm{m}^{2}\right.$ control left vs $0.579 \pm$ $0.072 \mu \mathrm{m}^{2}$ anastomosed right arteries $)(\mathrm{p}=0.137)$ did not change in anastomosed right arteries in the placebo group respectively. Rosiglitazone treatment did not affect either the medial $\left(0.540 \pm 0.094 \mu \mathrm{m}^{2}\right.$ PBS group vs $0.435 \pm 0.038 \mu \mathrm{m}^{2}$ rosiglitazone group) $(\mathrm{p}=0.999)$ or luminal cross-sectional areas $\left(0.487 \pm 0.085 \mu \mathrm{m}^{2}\right.$ PBS group vs $0.545 \pm 0.074 \mu \mathrm{m}^{2}$ rosiglitazone group) $(\mathrm{p}=0.953)$ respectively in anastomosed right arteries.

\section{Immunohistochemistry}

Immunoscoring with MMP-2 and MMP-9 antibodies revealed that immunopositivity for MMP-2 and MMP-9 significantly increased in anastomosed right arteries compared to control left arteries from placebo group (Figure 5, Table 1). However, immunoscoring for MMP-2 and MMP-9 showed that rosiglitazone treatment significantly decreased immunopositivity for these two proteins in anastomosed right carotid arteries compared to placebo group (Figure 6, Table 1).

\section{Discussion}

The primary reason for late bypass graft failure is neointimal hyperplasia which occurs in response to vascular injury $[18,19]$. In the present study we showed that carotid artery anastomosis model leads to intensive neointimal hyperplasia and increased intima/media index in rabbits from placebo group consistent with our previous findings [20]. However, luminal and medial areas remained unchanged in anastomosed arteries compared to control arteries from placebo group as we have observed previously in our study [20]. This latter finding may suggest that there is a expansive vascular remodeling to compensate narrowed luminal area and to maintain luminal patency in anastomosed arteries. Vascular 


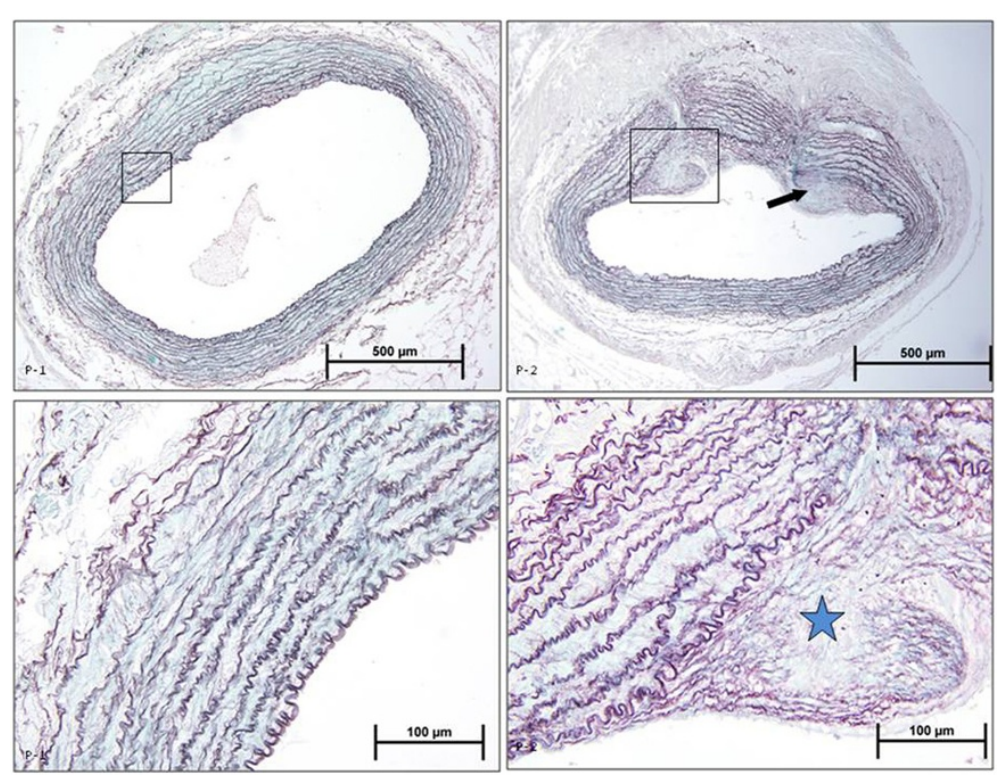

Figure 1 Photomicrographs of orcein light green stained rabbit carotid arteries. P1: Control left artery from PBS group, P2: Anastomosed right artery from PBS group. Star and arrow indicate intimal hyperplasia in the artery.

remodeling is an adaptive changing and healing process in arterial size and composition and also determines the degree of luminal narrowing in vascular occlusive diseases, restenosis and atherosclerosis [21,22]. Expansive vascular remodeling notably occurs during the early period of graft adaptation and protects luminal area [23]. However, we have not aimed to examine remodeling process in the arteries in this study. This point needs further investigation.

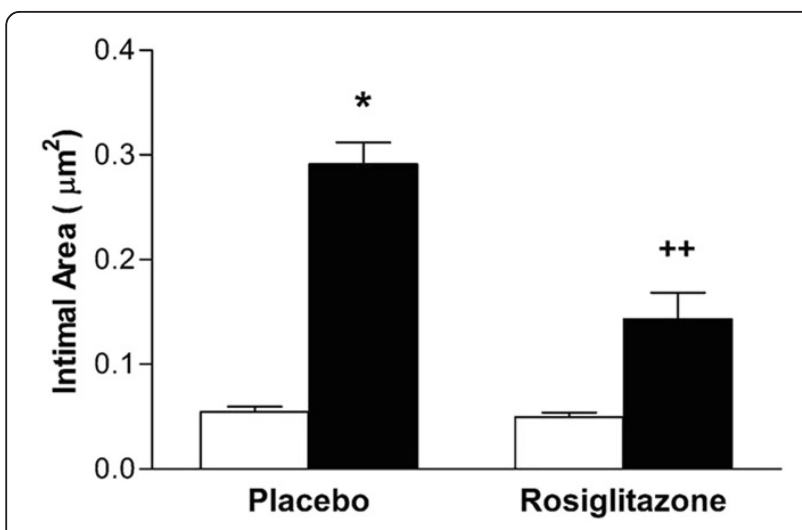

Figure 2 Effects of anastomosis and rosiglitazone on intimal hyperplasia in rabbit carotid arteries. White columns represent control left, black columns represent anastomosed right carotid arteries. $n$ represents number of the rabbits. Placebo group $(n=7)$, rosiglitazone group $(n=6)$. ${ }^{*} p<0.05$ Wilcoxon signed ranks test (control left vs anastomosed right arteries) and $++p<0.01$ Mann Whitney $\mathrm{U}$ test (placebo vs rosiglitazone groups).
In the present study, we also demonstrated that rosiglitazone inhibited neointimal hyperplasia induced by carotid artery anastomosis model in rabbits without affecting luminal or medial area. Previous reports have pointed out that PPAR- $\gamma$ receptor agonists could prevent restenosis and atherosclerosis [14,24]. Similarly, in a recent study, PPAR- $\gamma$ receptor ligand rosiglitazone was shown to decrease neointima and increase luminal area of the vessels after balloon catheter injury in collesterolfed rabbits [25]. Besides, it was reported that rosiglitazone reduced inflammatory response and retarded neointima progression after vascular injury in experimental atherosclerosis models in mouse and rats [26]. Furthermore, rosiglitazone was demonstrated to protect from restenosis and atherosclerosis after vascular injury in diabetic animals, and after coronary stent location in diabetic patients [14,24,27]. In all these models, antiatherosclerotic effects of rosiglitazone seem to be pleiotropic effects independent of their effects on glucose and lipid metabolism [28]. However, the underlying mechanisms of these pleiotropic effects are not clearly known. Therefore, we investigated the possible role of gelatinases and effects of rosiglitazone in our experimental atherosclerosis model in the current study. As parallel to our previous findings, we demonstrated semiquantitatively that carotid artery anastomosis model in rabbits induces gelatinase upregulation associated with neointimal hyperplasia formation [20]. We have observed increasing trend of gelatinase expression in this model previously. Unlikely, in the present study, we determined 


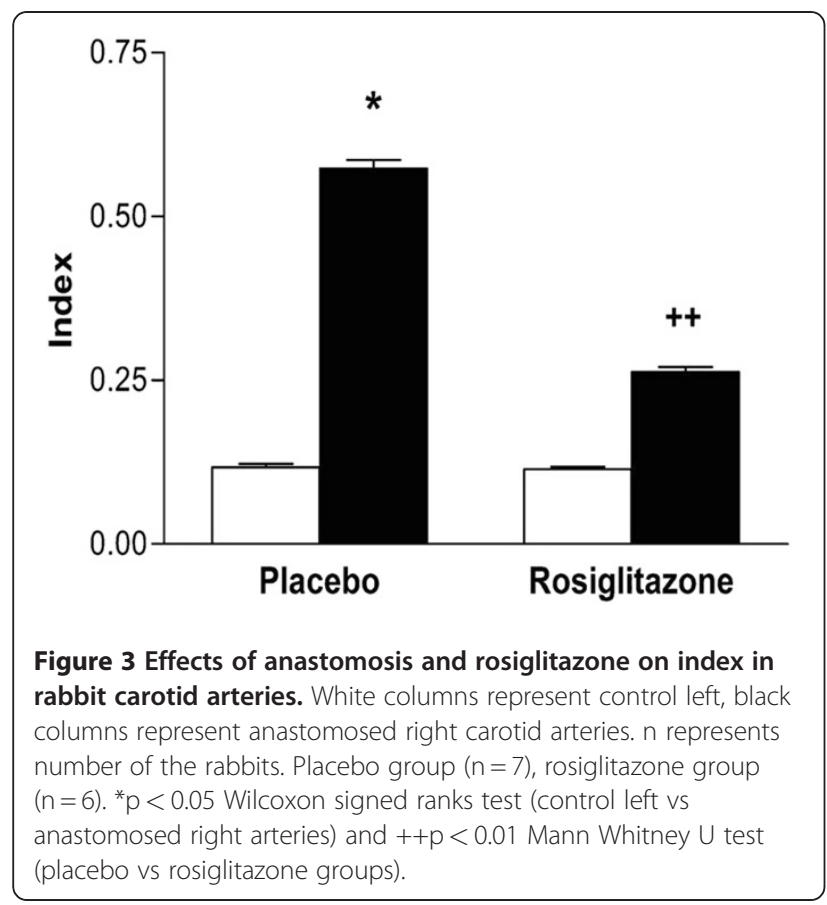

the increased expressions of two gelatinase enzymes semiquantitatively by scoring in rabbits [20].

Previous studies showed that unbalanced increase in MMP activity after vascular injury may cause various vascular pathologies including atherosclerosis, plaque rupture, restenosis and vein graft failure $[3,29,30]$. In particular, gelatinases (MMP-2 and MMP-9) were shown to be implicated in early atherosclerotic process [29,31]. It was reported that SMC migration from intima to media and induction of intimal hyperplasia substantially decreased in deficiency of gelatinases [32,33]. Consistent with our findings, recent studies demonstrated that gelatinases increased after vascular injury in femoral artery wire injury model in murins, and vascular graft or collar induced atherosclerosis models in rabbits [23,34,35]. Furthermore, increased gelatinase expression was shown in balloon-injured Sprague-Dawley rats and aortic smooth muscle cell culture, and also human saphenous vein organ culture from patients undergoing coronary bypass surgery [36,37]. Additionally, it was reported that administration of an MMP inhibitor after ballon injury or overexpression of TIMP-1 and TIMP-2 or inhibitors of gelatinases, respectively, inhibited neointima formation in vivo [38-40]. Our results support all these previous evidences concerning gelatinases and further underline the major role of gelatinases, and significance of ECM breakdown, promoting proliferation and migration of vascular SMCs in atherosclerotic process.

Additionally, the increase in the levels of pro-inflammatory gelatinases in the anastomosed arteries points out the presence of inflammation after vascular injury in our model. It was known that inflammatory cytokines such as interleukin-1 and -4 (IL-1 and IL-4) and tumour necrosis factor- $\alpha$ (TNF- $\alpha$ ) may coordinately induce gelatinase enzymes during progression of atherosclerosis [30]. Moreover, growth factors such as platellet derived growth factor (PDGF) and fibroblast growth factor-2 (FGF2) could interact with these cytokines [3]. Furthermore,
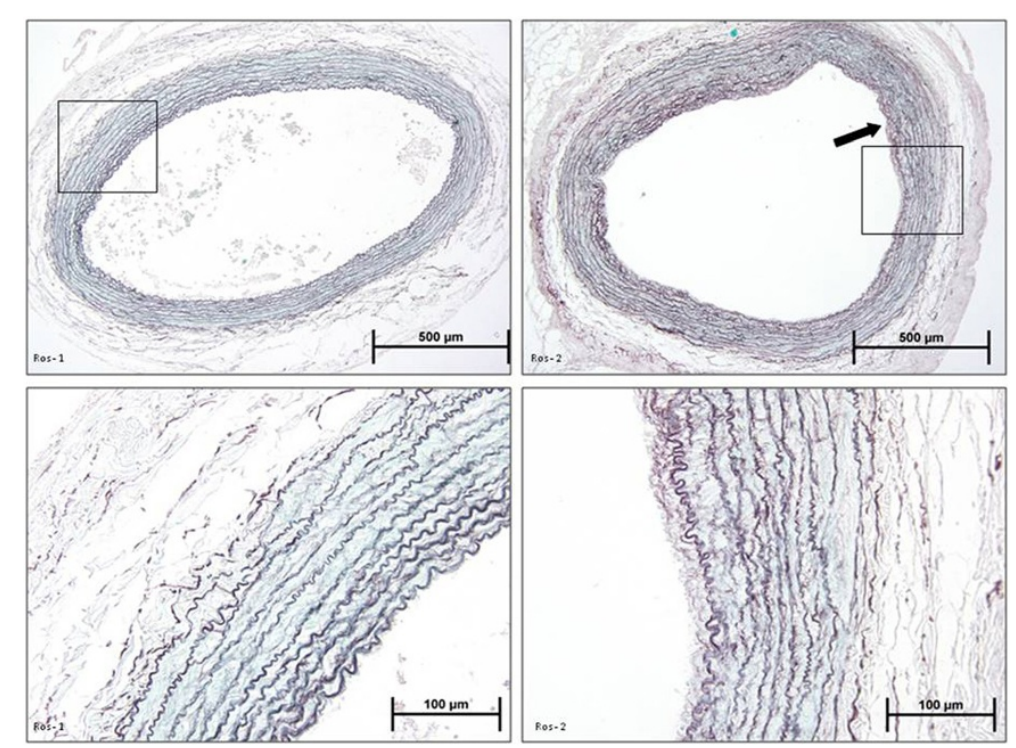

Figure 4 Photomicrographs of orcein light green stained rabbit carotid arteries. ROS1: Control left artery from rosiglitazone group, ROS2: Anastomosed right artery from rosiglitazone group. Arrow indicates intimal hyperplasia in the artery. 


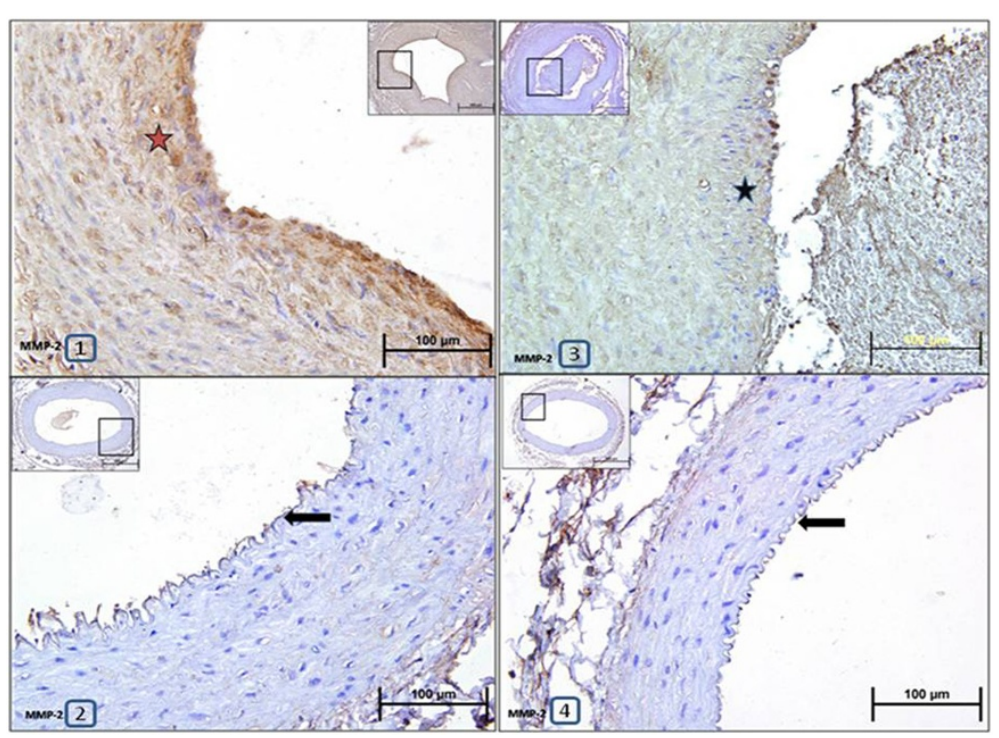

Figure 5 Representative photomicrographs of paraffin transverse sections of carotid arteries stained with MMP-2 antibody immunohistochemically. 1:Anastomosed right carotid artery from placebo group, 2:Control left carotid artery from placebo group, 3: Anastomosed right carotid artery from rosiglitazone-treated group, 4:Control left carotid artery from rosiglitazone-treated group. Stars show brown stained MMP-2 immunopositive areas in the arteries. Arrows point out intima layers of the arteries.

chemokines may direct transendotelial migration of monocytes and differentiation to macrophages. In the next step, monocytes and macrophages could cause increased MMP expression [41]. All these possible effects were shown to contribute to SMC proliferation and migration from intima to media layer, and finally intimal hyperplasia $[3,30]$. However, further studies are necessary to elucidate the underlying molecular signaling mechanisms of increased MMP expression in this model.

As another key finding, in the current study we have demonstrated for the first time that rosiglitazone

\begin{tabular}{|c|c|c|c|c|}
\hline Immunoscores & 0 & 1 & 2 & 3 \\
\hline \multicolumn{5}{|l|}{ MMP-2 } \\
\hline Placebo Left & 7 & 0 & 0 & 0 \\
\hline PlaceboRight* & 0 & 0 & 1 & 6 \\
\hline Rosiglitazon Left & 6 & 0 & 0 & 0 \\
\hline Rosiglitazon Right ++ & 0 & 4 & 2 & 0 \\
\hline \multicolumn{5}{|l|}{ MMP-9 } \\
\hline Placebo Left & 7 & 0 & 0 & 0 \\
\hline Placebo Right* & 0 & 0 & 2 & 5 \\
\hline Rosiglitazon Left & 6 & 0 & 0 & 0 \\
\hline Rosiglitazon Right ++ & 0 & 5 & 1 & 0 \\
\hline
\end{tabular}

significantly inhibited gelatinase expression in carotid artery anastomosis model in rabbits. Indeed, it was known that PPAR- $\gamma$ receptor agonists have important role in the modulation of vital processes such as inflammation, proliferation, differentiation and apoptosis independent of their actions in metabolic control [6,28]. In addition, Wu et al. demonstrated that LPS-induced gelatinase A (MMP-2) activity was inhibited by rosiglitazone in the rat aortic endothelial cells [42]. In agreement with this study, Lee et al. showed that rosiglitazone inhibited the expression and activity of gelatinase B (MMP-9), and vascular SMC proliferation and migration through GSK-3 $\beta$ activation and inhibition of NF-kB and ERK pathways in rat aortic vascular SMC culture [13]. Furthermore, Rinaldi et al. showed that rosiglitazone diminished neointimal hyperplasia and inflammatory events by reducing numbers and interactions of inflammatory cells and also negatively regulating the activations of inflammatory markers such as p38 MAPK, NF-B, COX-2, and HSP 47 [26]. Besides, Ling et al. demonstrated that rosiglitazone significantly decreased MMP-2 mRNA expression and activity, and inhibited VSMC proliferation in cultured VSMCs which were isolated from rat thoracic aortas [43]. Evidence from clinical studies are similar to the data from numerous animal studies. Clinical studies showed that patients with a history of coronary artery disease (CAD) and type 2 diabetes had significantly elevated serum MMP-9 levels $[44,45]$. High levels of MMP-9 were reported to trigger plaque instability and rupture [46]. However, rosiglitazone treatment decreased MMP-9 plasma levels in patients 


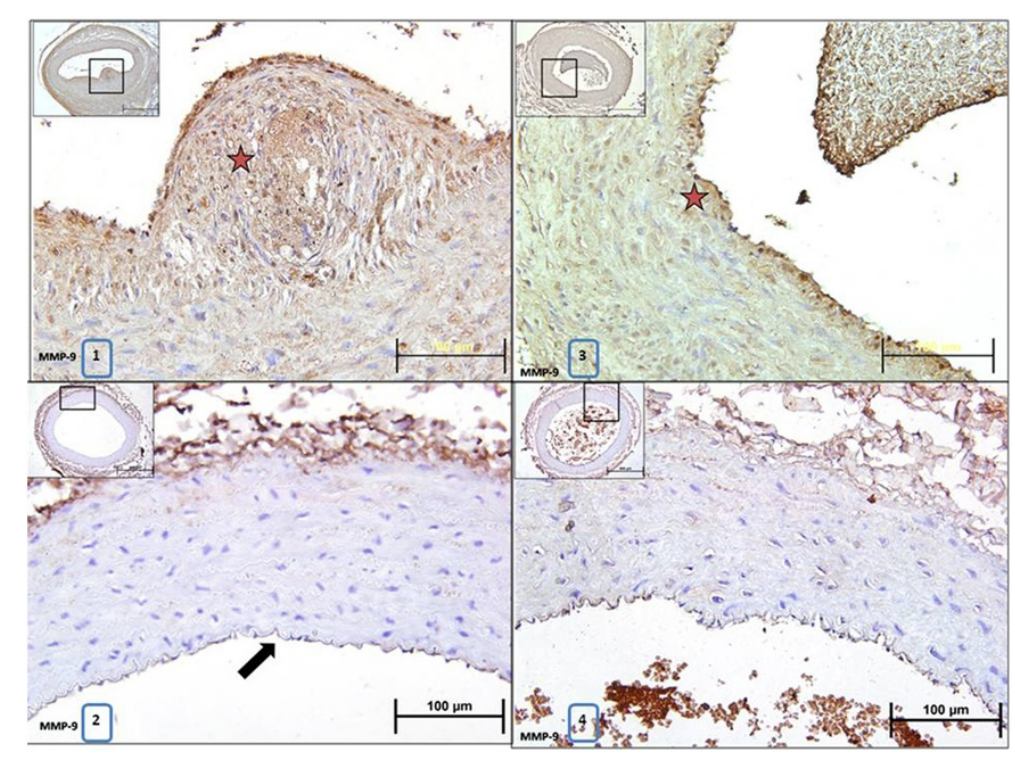

Figure 6 Representative photomicrographs of paraffin transverse sections of carotid arteries stained with MMP-9 antibody

immunohistochemically. 1:Anastomosed right carotid artery from placebo group, 2:Control left carotid artery from placebo group, 3 : Anastomosed right carotid artery from rosiglitazone-treated group, 4:Control left carotid artery from rosiglitazone-treated group. Stars show brown stained MMP-9 immunopositive areas in the arteries. Arrow points out intima layer of the artery.

with CAD and diabetes [45]. Overexpression of MMP-9 in the human vulnerable plaques indicates the crucial role of MMP-9 in patients with acute coronary syndrome [46]. Therefore, the inhibitory effect of rosiglitazone on MMP-9 found in the present study may contribute to beneficial effects of rosiglitazone on cardiovascular risk of the patients with previous acute myocardial infarction.

\section{Conclusion}

The present study demonstrated that PPAR- $\gamma$ agonist rosiglitazone inhibited neointimal hyperplasia and expression of proinflammatory MMP enzymes, which have a crucial role in initiation and progression of atherosclerosis or restenosis in carotid artery anastomosis model in rabbits.

Our results suggest that rosiglitazone may play an atheroprotective role via its beneficial effects on gelatinases (MMP-2 and -9) throughout inflammatory process particularly in the high cardiovascular risk population of patients with diabetes mellitus. Even though some adverse effects of rosiglitazone treatment have been reported recently, there is insufficient evidence of any adverse effect on developing cardiovascular disease. Therefore, it is still used in USA with approval of FDA. However, further studies are necessary to elucidate the molecular mechanism of PPAR $\gamma$ agonists in vascular protection. Collected data will be useful to prove that future PPAR agonists not only prevent atherosclerotic events but also result in a net reduction on total cardiovascular incidents without any significant noncardiovascular adverse effects with long-term use.

\section{Competing interests}

The authors declare that they have no competing interests.

\section{Authors' contribution}

$M G$, carried out the molecular genetic studies. BR, carried out the molecular genetic studies. SA, participated in the sequence alignment and drafted the manuscript. $A B$, participated in the sequence alignment and drafted the manuscript. EH participated in the sequence alignment and drafted the manuscript. All authors read and approved the final manuscript.

\section{Acknowledgement}

This study was supported by Mustafa Kemal University Research Fund (Project No: 02 M 104 to M.G).

\section{Author details}

'Department of Cardiovascular Surgery, Faculty of Medicine, Izmir University, Izmir, Turkey. ${ }^{2}$ Department of Pharmacology, Faculty of Pharmacy, Ege University, Izmir, Turkey. ${ }^{3}$ Department of Histology and Embriology, Faculty of Medicine, Dokuz Eylul University, Izmir, Turkey.

Received: 22 February 2012 Accepted: 8 June 2012

Published: 20 June 2012

\section{References}

1. Libby $\mathrm{P}$, Tanaka $\mathrm{H}$ : The molecular bases of restenosis. Prog Cardiovasc Dis 1997, 40(2):97-106.

2. O' Brien ER, Ma X, Simard T, Pourdjabbar A, Hibbert B: Pathogenesis of Neointima formation following vascular injury. Cardiovasc Hematol Disord Drug Targets 2011, 11(1):30-39.

3. Newby AC: Matrix metalloproteinases regulate migration, proliferation, and death of vascular smooth muscle cells by degrading matrix and non-matrix substrates. Cardiovasc Res 2006, 69(3):614-624.

4. Ricote M, Huang J, Fajas L, Li A, Welch J, Najib J, et al: Expression of the peroxisome proliferator-activated receptor gamma (PPARgamma) in human atherosclerosis and regulation in macrophages by colony stimulating factors and oxidized low density lipoprotein. Proc Natl Acad Sci USA 1998, 95(13):7614-7619. 
5. Law RE, Goetze S, Xi XP, Jackson S, Kawano Y, Demer L, et al: Expression and function of PPARgamma in rat and human vascular smooth muscle cells. Circulation 2000, 101(11):1311-1318.

6. van Bilsen $M$, van Nieuwenhoven FA: PPARs as therapeutic targets in cardiovascular disease. Expert Opin Ther Targets 2010, 14(10):1029-1045.

7. Schiffrin EL: Peroxisome proliferator-activated receptors and cardiovascular remodeling. Am J Physiol Heart Circ Physiol 2005, 288(3):H1037-H1043.

8. Yiqin $Y$, Meilin X, Jie X, Keping Z: Aspirin inhibits MMP-2 and MMP-9 expression and activity through PPARalpha/gamma and TIMP-1mediated mechanisms in cultured mouse celiac macrophages. Inflammation 2009, 32(4):233-241.

9. Chang K, Francis SA, Aikawa E, Figueiredo JL, Kohler RH, McCarthy JR, et al: Pioglitazone suppresses inflammation in vivo in murine carotid atherosclerosis: novel detection by dual-target fluorescence molecular imaging. Arterioscler Thromb Vasc Biol 2010, 30(10):1933-1939.

10. Meredith D, Panchatcharam M, Miriyala S, Tsai YS, Morris AJ, Maeda N, et al: Dominant-negative loss of PPARgamma function enhances smooth muscle cell proliferation, migration, and vascular remodeling. Arterioscler Thromb Vasc Biol 2009, 29(4):465-471.

11. Robinson E, Grieve DJ: Significance of peroxisome proliferator-activated receptors in the cardiovascular system in health and disease. Pharmacol Ther 2009, 122(3):246-263.

12. Zahradka P, Wright B, Fuerst M, Yurkova N, Molnar K, Taylor CG: Peroxisome proliferator-activated receptor alpha and gamma ligands differentially affect smooth muscle cell proliferation and migration. J Pharmacol Exp Ther 2006, 317(2):651-659.

13. Lee CS, Kwon YW, Yang HM, Kim SH, Kim TY, Hur J, et al: New mechanism of rosiglitazone to reduce neointimal hyperplasia: activation of glycogen synthase kinase-3beta followed by inhibition of MMP-9. Arterioscler Thromb Vasc Biol 2009, 29(4):472-479.

14. Choi D, Kim SK, Choi SH, Ko YG, Ahn CW, Jang Y, et al: Preventative effects of rosiglitazone on restenosis after coronary stent implantation in patients with type 2 diabetes. Diabetes Care 2004, 27:2654-2660

15. Fang CC, Ng Jao, YT Yi-Chen, Yu CL, Chen CL, Wang SP: Angiographic and clinical outcomes of rosiglitazone in patients with type 2 diabetes mellitus after percutaneous coronary interventions: a single center experience. Angiology 2007, 58(5):523-534.

16. Alessi A, França Neto OR, Brofman PR, Prim C, Noronha L, Silva RF, et al: Use of rosiglitazone before and after vascular injury in hypercholesterolemic rabbits: Assessment of neointimal formation. Thromb J 2008, 6:12.

17. Freire Cerqueira N, Hussni CA, Bonetti Yoshida W, Swain Müller S, Sequeira $J$, Rodrigues $A C$, et al: Effect of diclofenac sodium on the healing process of end-to-end anastomosis in carotid arteries of rabbits.

Histopathological and biomechanical studies. Int Angio/ 2003, 22(4):431-437.

18. De Meyer GR, Bult H: Mechanisms of neointima formation -lessons from experimental models. Vasc Med 1997, 2(3):179-189.

19. Jackson MR, Belott TP, Dickason T, Kaiser WJ, Modrall JG, Valentine RJ, et al: The consequences of a failed femoropopliteal bypass grafting: comparison of saphenous vein and PTFE grafts. J Vasc Surg 2000, 32(3):498-504. 504-5

20. Güzeloğlu M, Gül M, Reel B, Yürekli I, Aykut K, Hazan E: The effects of zoledronic acid on neointimal hyperplasia: a rabbit carotid anastomosis model. Anadolu Kardiyol Derg 2011, 11(2):93-100.

21. Sluijter JP, de Kleijn DP, Pasterkamp G: Vascular remodeling and protease inhibition-bench to bedside. Cardiovasc Res 2006, 69(3):595-603.

22. Mitchell RN, Libby P: Vascular remodeling in transplant vasculopathy. Circ Res 2007, 100(7):967-978

23. Wong AP, Nili N, Jackson ZS, Qiang B, Leong-Poi $H$, Jaffe $R$, et al: Expansive remodeling in venous bypass grafts: novel implications for vein graft disease. Atherosclerosis 2008, 196(2):580-589.

24. Phillips JW, Barringhaus KG, Sanders JM, Yang Z, Chen M, Hesselbacher S, et al: Rosiglitazone reduces the accelerated neointima formation after arterial injury in a mouse injury model of type 2 diabetes. Circulation 2003, 108:1994-1999.

25. Alessi A, França Neto OR, Prim C, Silva RF, Noronha L, Brofman PR, et al: Rosiglitazone and vascular injury in hypercholesterolemic rabbits: neointimal formation assessment. Arq Bras Cardiol 2010, 95(3):283-288.

26. Rinaldi B, Pieri L, Donniacuo M, Cappetta D, Capuano A, Domenici L, et al: Rosiglitazone reduces the inflammatory response in a model of vascular injury in rats. Shock 2009, 32(6):638-644.

27. Desouza CV, Murthy SN, Diez J, Dunne B, Matta AS, Fonseca VA, et al: Differential effects of peroxisome proliferator activator receptor-alpha and gamma ligands on intimal hyperplasia after balloon catheter- induced vascular injury in Zucker rats. J Cardiovasc Pharmacol Ther 2003, 8:297-305.

28. Salomone S: Pleiotropic effects of glitazones: a double edge sword? Front Pharmacol 2011, 2(14):1-6.

29. Galis ZS, Johnson C, Godin D, Magid R, Shipley JM, Senior RM, et al: Targeted disruption of the matrix metalloproteinase- 9 gene impairs smooth muscle cell migration and geometrical arterial remodeling. Circ Res 2002, 91(9):852-859.

30. Newby AC: Dual role of matrix metalloproteinases (matrixins) in intimal thickening and atherosclerotic plaque rupture. Physiol Rev 2005, 85(1):1-31.

31. Johnson C, Galis ZS: Matrix metalloproteinase-2 and -9 differentially regulate smooth muscle cell migration and cell-mediated collagen organization. Arterioscler Thromb Vasc Biol 2004, 24(1):54-60.

32. Cho A, Reidy MA: Matrix metalloproteinase- 9 is necessary for the regulation of smooth muscle cell replication and migration after arterial injury. Circ Res 2002, 91(9):845-851.

33. Kuzuya M, Kanda S, Sasaki T, Tamaya-Mori N, Cheng XW, Itoh T, et al: Deficiency of gelatinase a suppresses smooth muscle cell invasion and development of experimental intimal hyperplasia. Circulation 2003, 108(11):1375-1381.

34. Zou Y, Qi Y, Roztocil E, Davies MG: Patterns of gelatinase activation induced by injury in the murine femoral artery. J Surg Res 2009, 154(1):135-142.

35. Reel B, Oktay G, Ozkal S, Islekel H, Ozer E, Ozsarlak-Sozer G, et al: MMP-2 and MMP-9 alteration in response to collaring in rabbits: the effects of endothelin receptor antagonism. J Cardiovasc Pharmacol Ther 2009, 14(4):292-301.

36. Kranzhöfer A, Baker AH, George SJ, Newby AC: Expression of tissue inhibitor of metalloproteinase-1, -2 , and -3 during neointima formation in organ cultures of human saphenous vein. Arterioscler Thromb Vasc Biol 1999, 19(2):255-265.

37. Chang W, Lim S, Song H, Song BW, Kim HJ, Cha MJ, et al: Cordycepin inhibits vascular smooth muscle cell proliferation. Eur J Pharmacol 2008, 597(1-3):64-69.

38. Bendeck MP, Zempo N, Clowes AW, Galardy RE, Reidy MA: Smooth muscle cell migration and matrix metalloproteinase expression after arterial injury in the rat. Circ Res 1994, 75(3):539-545.

39. Forough R, Koyama N, Hasenstab D, Lea H, Clowes M, Nikkari ST, et al: Overexpression of tissue inhibitor of matrix metalloproteinase- 1 inhibits vascular smooth muscle cell functions in vitro and in vivo. Circ Res 1996 79(4):812-820

40. Cheng L, Mantile G, Pauly R, Nater C, Felici A, Monticone R, et al: Adenovirus-mediated gene transfer of the human tissue inhibitor of metalloproteinase-2 blocks vascular smooth muscle cell invasiveness in vitro and modulates neointimal development in vivo. Circulation 1998, 98(20):2195-2201.

41. Reel B, Sala-Newby GB, Huang WC, Newby AC, et al: Diverse patterns of cyclo-oxygenase-independent metalloproteinase gene regulation in human monocytes. Br J Pharmacol 2011, doi:10.1111/j.14765381.2011.01298.x

42. Wu X, Li L: Rosiglitazone suppresses lipopolysaccharide-induced matrix metalloproteinase-2 activity in rat aortic endothelial cells via Ras-MEK1/2 signaling. Int J Cardiol 2011, Epub ahead of print.

43. Ling HY, Hu B, Wang BX, Zu XY, Feng SD, Ou HS, et al: Effects of rosiglitazone on the proliferation of vascular smooth muscle cell induced by high glucose. Cardiovasc Drugs Ther 2008, 22(6):453-460.

44. Ferroni $P$, Basili S, Martini F, Cardarello CM, Ceci F, Di Franco M, et al: Serum metalloproteinase 9 levels in patients with coronary artery disease: a novel marker of inflammation. J Investig Med 2003, 51 (5):295-300.

45. Marx N, Froehlich J, Siam L, Ittner J, Wierse G, Schmidt A, et al: Antidiabetic PPAR gamma-activator rosiglitazone reduces MMP- 9 serum levels in type 2 diabetic patients with coronary artery disease. Arterioscler Thromb Vasc Biol 2003, 23(2):283-288.

46. Newby AC: Do metalloproteinases destabilize vulnerable atherosclerotic plaques? Curr Opin Lipidol 2006, 17(5):556-561.

doi:10.1186/1749-8090-7-57

Cite this article as: Guzeloglu et al:: The effects of PPAR $y$ agonist rosiglitazone on neointimal hyperplasia in rabbit carotid anastomosis model. Journal of Cardiothoracic Surgery 2012 7:57. 